\section{UCDNN}

LIBRARY
University of Connecticut OpenCommons@UConn

June 1993

\title{
Evaluation of Root Resorption Incident to Orthodontic Intrusion
}

Greg Costopoulos

Follow this and additional works at: https://opencommons.uconn.edu/sodm_masters

\section{Recommended Citation}

Costopoulos, Greg, "Evaluation of Root Resorption Incident to Orthodontic Intrusion" (1993). SoDM Masters Theses. 29.

https://opencommons.uconn.edu/sodm_masters/29 


\title{
An evaluation of root resorption incident to orthodontic intrusion.
}

\author{
by \\ Greg Costopoulos, D.D.S, M.S. \\ In partial fulfillment of the requirements for a \\ Certificate in Orthodontics
}

Major advisor: Ravindra Nanda, B.D.S., M.D.S., Ph.D. Co-advisor: Andrew Kuhlberg, D.M.D., M.S.D.

Department of Orthodontics

University of Connecticut, School of Dental Medicine 


\section{APPROVAL PAGE}

An evaluation of root resorption incident to orthodontic intrusion.

by Greg Costopoulos, D.D.S., MS.

Major advisor:

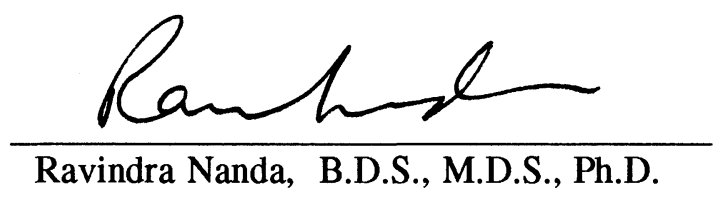

Ravindra Nanda, B.D.S., M.D.S., Ph.D.

Co-advisor:

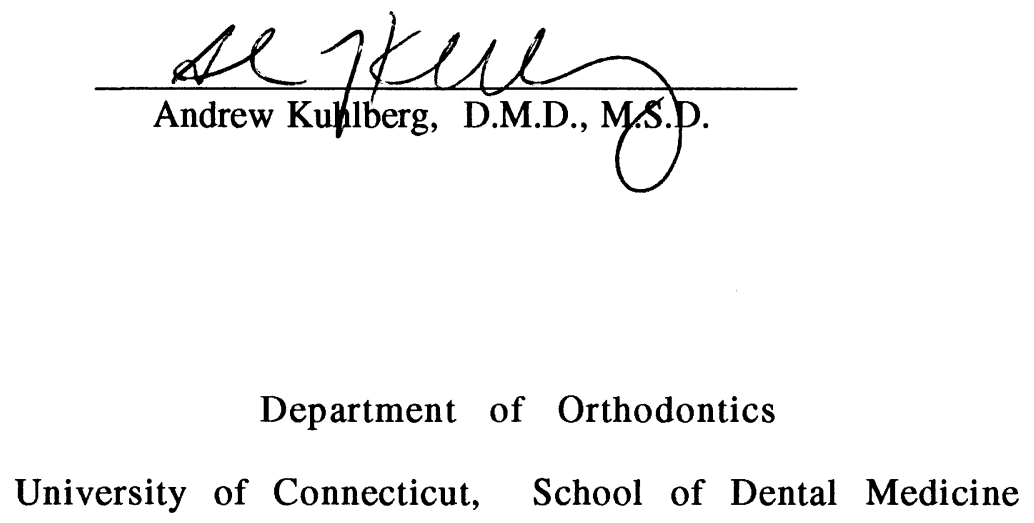




\section{Abstract}

A new radiographic method was developed for measuring changes in root length. Using this technique, orthodontic intrusion as a potential cause of apical root resorption of maxillary incisors was investigated. The experimental group consisted of 17 patients with excessive overbite who were treated using a Burstone type intrusion arch delivering a low level of force (about $15 \mathrm{gm}$ per tooth). A control group was made up of 17 patients in full arch fixed appliances who were randomly selected. After a period of approximately 4 months the intrusion group had only slightly more root resorption than the controls, $0.6 \mathrm{~mm}$ vs. $0.2 \mathrm{~mm}$ (statistically signifcant difference). Intrusion measured at the center of resistance of the central incisor averaged $1.9 \mathrm{~mm}$. The amount of resorption was not correlated with the amount of intrusion. A moderate correlation, $r=0.45$, was found between resorption and movement of the apex (i.e. in addition to intrusion there was often lingual or facial root movement). Results of this study indicate that intrusion with low forces can be effective in reducing overbite while causing only a negligible amount of apical root resorption. 


\section{Introduction}

Some degree of apical root resorption is frequently a side effect of orthodontic tooth movement. Early investigators of this phenomenon such as Ketcham(1) and Phillips(2) found maxillary incisors to be the most susceptible. At this time, evidence indicates that a routine course of orthodontic treatment will lead to an average apical resorption of 1 to 2 millimeters $(\mathrm{mm})$ for upper incisors $(3,4,5,6,7,8)$ with 2 - 3\% of patients showing a loss of $4 \mathrm{~mm}$ or more $(4,7,9)$.

Intrusion is one of the specific types of tooth movement which has been suggested as a possible cause of root resorption $(10,11,12)$. The tooth apex and associated periodontium can experience relatively high compression stresses when an intrusive force is applied to the crown(13). Because of the potential for these high stress levels, intrusion is a technique which logically could increase the risk of apical root resorption.

Several investigators have examined the relationship between intrusion of incisors and root resorption. Deshields(3) attempted to correlate movement of the upper central incisors with root resorption. Using pre- and post-treatment lateral cephalograms he measured vertical tooth movement relative to a palatal plane, including correcting for normal growth. He found no correlation between intrusion and resorption. In a similar study by Kaley and Phillips(6), records of 200 consecutively treated patients were examined. These authors also failed to see a correlation between root resorption and net intrusive movement of maxillary or mandibular incisors. They did however, find a 20 fold increase in risk for severe resorption if the roots were moved in proximity to the lingual or facial cortical plates. 
McFadden et al.(17) looked at retrospective data of 38 deep bite patients. Mean treatment time was 29 months and included use of a utility arch exerting 20-25 gm per maxillary incisor. Measured relative to the palatal plane, the average amount of intrusion was $-0.8 \mathrm{~mm}$ or a net extrusion (but less than the normal increase in denture height of untreated individuals). Root shortening averaged $1.8 \mathrm{~mm}$ per tooth with no significant correlation between resorption and the amount of intrusion. Total treatment time was significantly correlated to root resorption. The authors conclude that the effect of the utility arch amounts to "holding against growth" and is unrelated to root shortening.

In a prospective study by Goerigk et al.(15), 31 patients were treated with an intrusion arch as described by Burstone(16). Lateral cephalograms and periapicals were taken before and after the intrusion phase of treatment (mean of 4.3 months). The authors defined intrusion in the maxillary arch as vertical change of the incisal edge perpendicular to the palatal plane. Average intrusion for the maxillary incisors was $2.3 \mathrm{~mm}$. The average amount of root resorption at the completion of intrusion was found to be $1.0 \mathrm{~mm}$.

A.study specifically designed to examine orthodontic intrusion as a possible cause of apical resorption of upper incisors was published by Dermaut and De Munck(10). 20 patients were treated using a Burstone(16) type intrusion arch for an average of 6.7 months. Intrusive force levels were regulated at $25 \mathrm{gm}$ per tooth. A control group consisted of 15 untreated subjects with two sets of periapical radiographs taken at an interval of 6.5 months. Findings for the experimental group included an average intrusion of $3.6 \mathrm{~mm}$ (measured as the vertical movement of the center of resistance of the central incisor). The mean loss of root length was $18 \%$ or $2.9 \mathrm{~mm}$. The control group served as a check of the reliability of the method and, as would be 
expected, showed virtually no change in root length. The authors did not find a significant correlation between the amount of resorption and the amount or duration of intrusion.

Assessing the extent of root resorption has often been done by visual examination and qualitative evaluation of periapical radiographs $(4,7,17,18,19)$. A more quantitative method described by Linge and Linge(8) is now more or less the standard. The length of the root from cementoenamel junction (CEJ) to apex is measured on pre- and post-treatment periapicals. Any change in root length is corrected for radiographic magnification differences using a ratio between crown lengths in the before and after films. This method is reasonably accurate but has some weaknesses. It relies on locating the CEJ which can be very susceptible to interpretation by the observer. Also, because the CEJ changes height moving circumferentially around the tooth, variation in x-ray beam angulation with respect to the tooth will project the CEJ differently.

The technical difficulties involved in quantifying resorption, as well as inconsistencies in results of previous clinical studies, make it unclear as to how large a role intrusion may play in causing root resorption. In light of this uncertainty, the present study sought to do the following:

1) Develop a highly accurate technique for quantifying apical root resorption.

2) Assess the amount of apical root resorption caused by an intrusive force of low magnitude on maxillary incisors.

3) Investigate the relationship between duration of the use of intrusion mechanics and the extent of root resorption.

4) Investigate the relationship between the amount of tooth movement and the extent of root resorption. 
It was hypothesized that intrusion with an appliance which can be reliably calibrated to deliver a low level of intrusive force will not cause a significant amount of root resorption.

\section{Materials and Methods}

Subjects: An experimental and control group each consisted of 17 patients selected from those receiving treatment in the graduate orthodontic clinic of the dental scool. Inclusion criteria for both groups were: completed incisor root formation, no history of marked root resorption prior to orthodontic treatment as evidenced on periapical films, no history of major trauma to maxillary incisors, and no previous orthodontic treatment. Additionally, the experimental subjects had a treatment plan which called for $2.0-4.0 \mathrm{~mm}$ of intrusion of the maxillary incisors. Control subjects were randomly chosen from amongst those patients undergoing full arch fixed appliance therapy without utilization of an intrusion base arch. The mean ages for the control and experimental groups were 16.1 and 16.4 years respectively.

Intrusion mechanics: The appliance used for intrusion was constructed as described by Burstone(16). It has low a load/deflection rate and is capable of delivering a fairly constant level of intrusive force to the incisors without torque(20). The appliance consisted of a .017" x .025" TMA base arch from the auxiliary tube of first molar to first molar. Activation was done by placing a bend in the wire mesial to the molar. The active intrusion arch was tied to a rigid anterior segmental wire and cinched or tied back at the molar. Force levels were adjusted to approximately 15 gm per tooth and checked usually at each visit. Calibration was 
done in the mouth using a light force gauge. First molars were often consolidated with the premolars using a section of rigid wire adjusted to be passive. The right and left posterior segments were joined by a palatal bar for further anchorage. In five cases a "3 piece" intrusion arch was used because the incisors were excessively flared to begin with. This modification uses two cantilevers from the molars instead of a continuous base arch; additionally, a light retraction force is placed on the anterior segment with chain elastic.

Measurements: To measure changes in position of the central incisor (only for the experimental group), a lateral cephalometric radiograph was taken before and after the intrusion phase of treatment. On a small piece of acetate a tracing was made of the central incisor in the original head film . A longitudinal axis was drawn and also the center of resistance (CR) - estimated as the midpoint of the root. This tracing was left in place on the film, then on another sheet of acetate the palate and maxillary landmarks such as key ridges and third molars were traced along with the central incisor using the underlying first tracing as a "template". The template of the incisor was then placed over the central incisor in the post-intrusion cephalogram. The maxillary tracing from the first radiograph was superimposed on the maxilla in the new film and the template of the incisor was traced - showing the net change in its position. The following characteristics of the movement of the central incisor were measured: intrusion (defined as vertical movement of CR), vertical change of the incisal edge, change in proclination, anteroposterior change of the incisal edge, and linear movement of the apex(without respect to direction) (see Figure 1) 
1) intrusion

2) vertical change of incisal edge

3) proclination

4) AP change of incisal edge

5) movement of apex

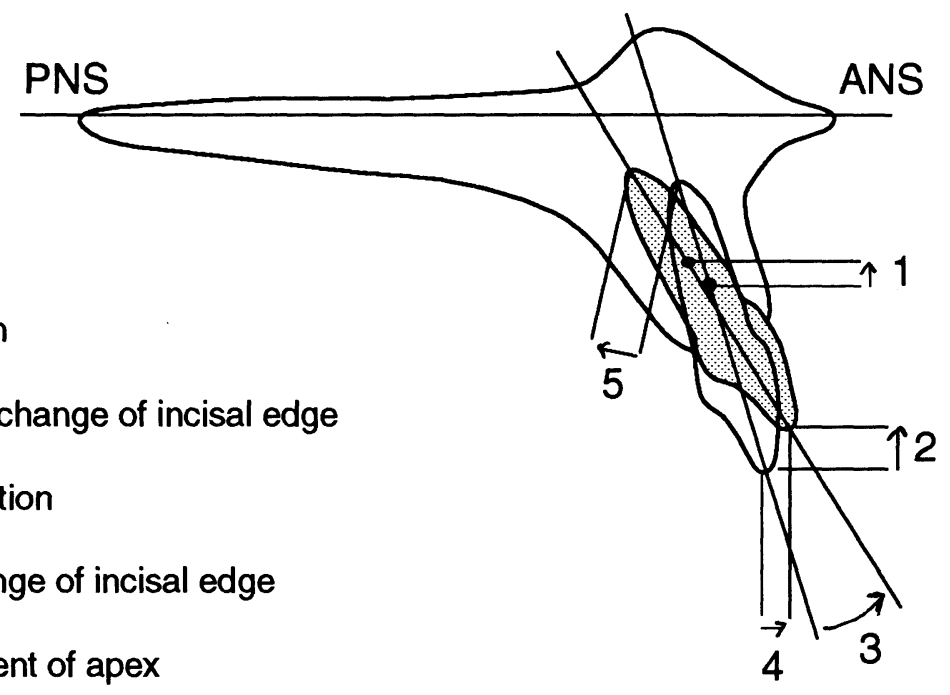

Figure 1 . Measurements of central incisor movement. 
To measure root resorption, periapical radiographs were taken before and after intrusion for the experimental subjects. The control subjects had periapical films taken at two points in time with the interval matched to the average time between films for the experimental group. All periapicals were taken with a special jig temporarily ligated to one of the central incisors. This device consists of a small acrylic block custom made to conform to the incisal edge and lingual surface of the tooth. The acrylic supports a section of .030" stainless steel wire, approximately 17 $\mathrm{mm}$ long, in front of the labial surface of the tooth and parallel to the long axis. A special "lateral periapical" radiograph taken with the jig in place to allows for adjusting the wire to make it parallel with the root. A standard periapical was then taken which results in a shadow of the wire superimposed on the image of the tooth The following series of photographs illustrates clinical aspects of the technique: (Figures 2 - 8). 


\section{Figure 2.}

The wire element, made from .030" stainless steel, is positioned on the patient's plaster model using soft wax. An attempt is made to have the vertical part parallel to the long axis of the central incisor. Cold cure acrylic is flowed over the horizontal part of the wire as well as the lingual surface and incisal edge of the tooth. After the acrylic cures, the wire can be slid out and back in to adjust as needed for clearance from the gingiva.

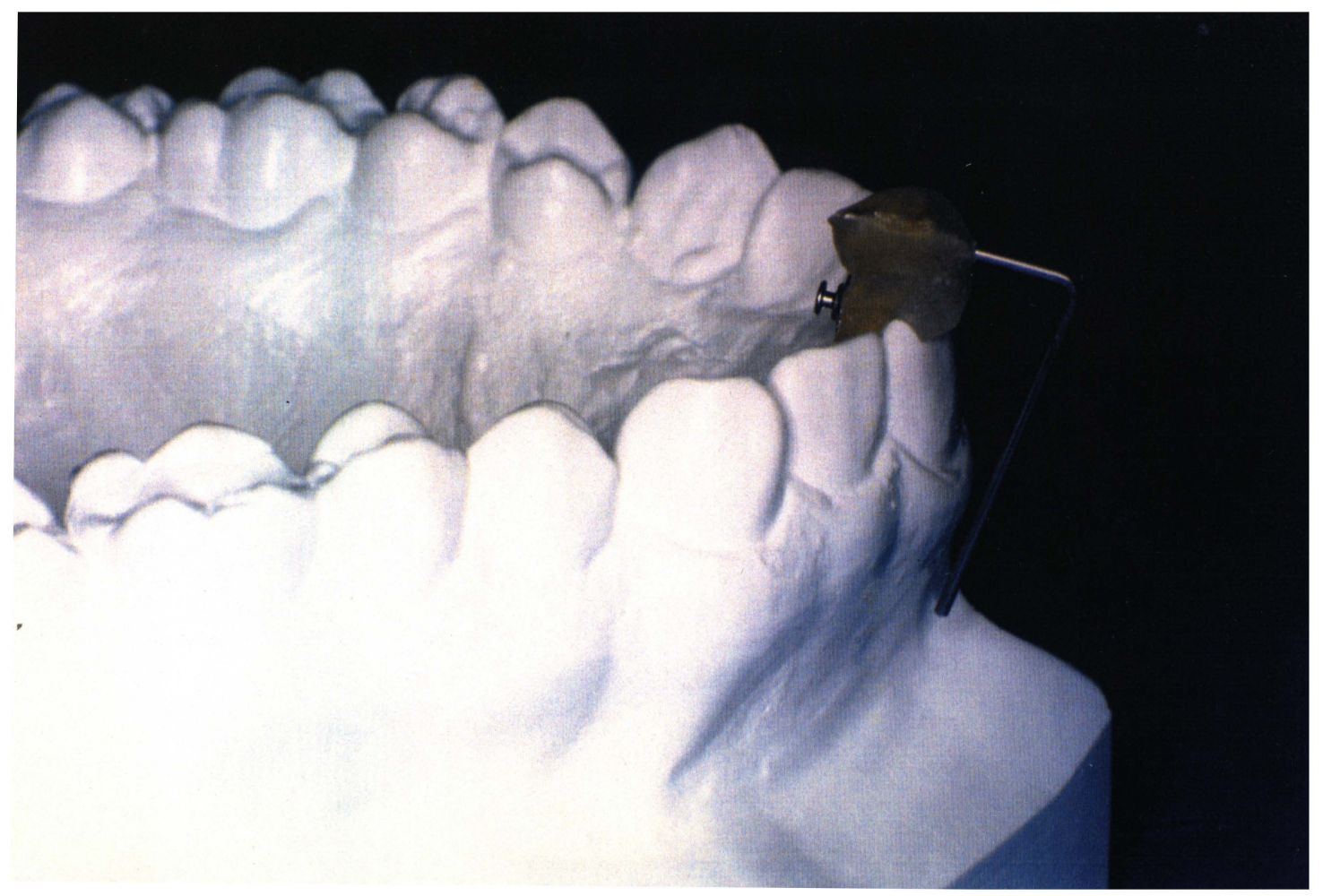




\section{Figure 3.}

A close up photograph of the jig. The wire part is fabricated from two sections of .030 stainless steel approximately $17 \mathrm{~mm}$ long. After rounding off the ends one piece is bent $3 \mathrm{~mm}$ from the end to about $80^{\circ}$. The short leg is welded to the straight piece of wire which will serve in calculating radiographic magnification. Two lingual buttons are attached with cold cure acrylic to facilitate ligation to the tooth.

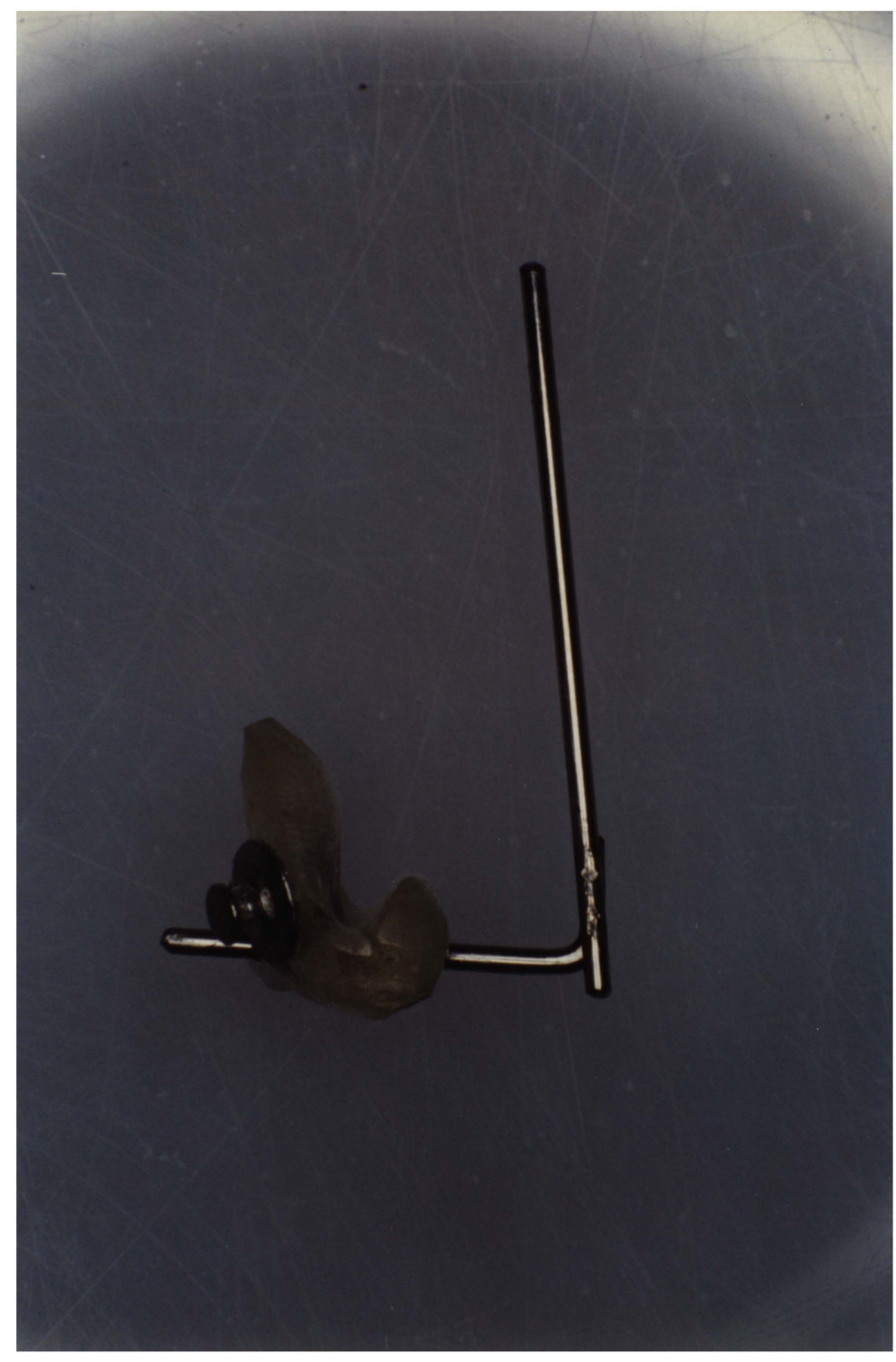




\section{Figure 4.}

The device is ligated to the patient's tooth (chain elastic works well for this).

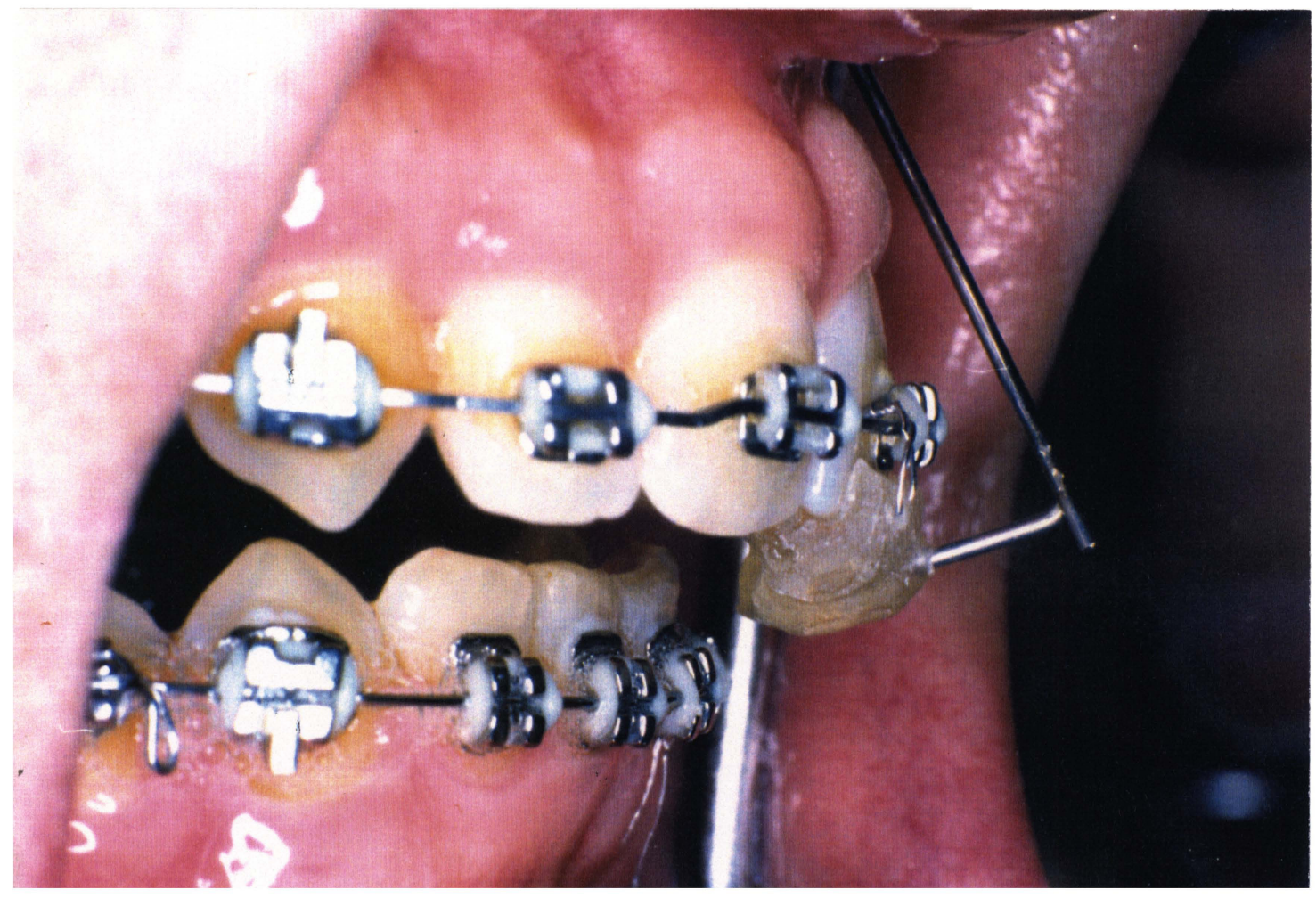




\section{Figure 5.}

Patient with the jig in place and set up for a "lateral periapical" radiograph using a styrofoam film holder attached to a tongue depressor. Occlusal size $\mathrm{x}$-ray film is preferable.

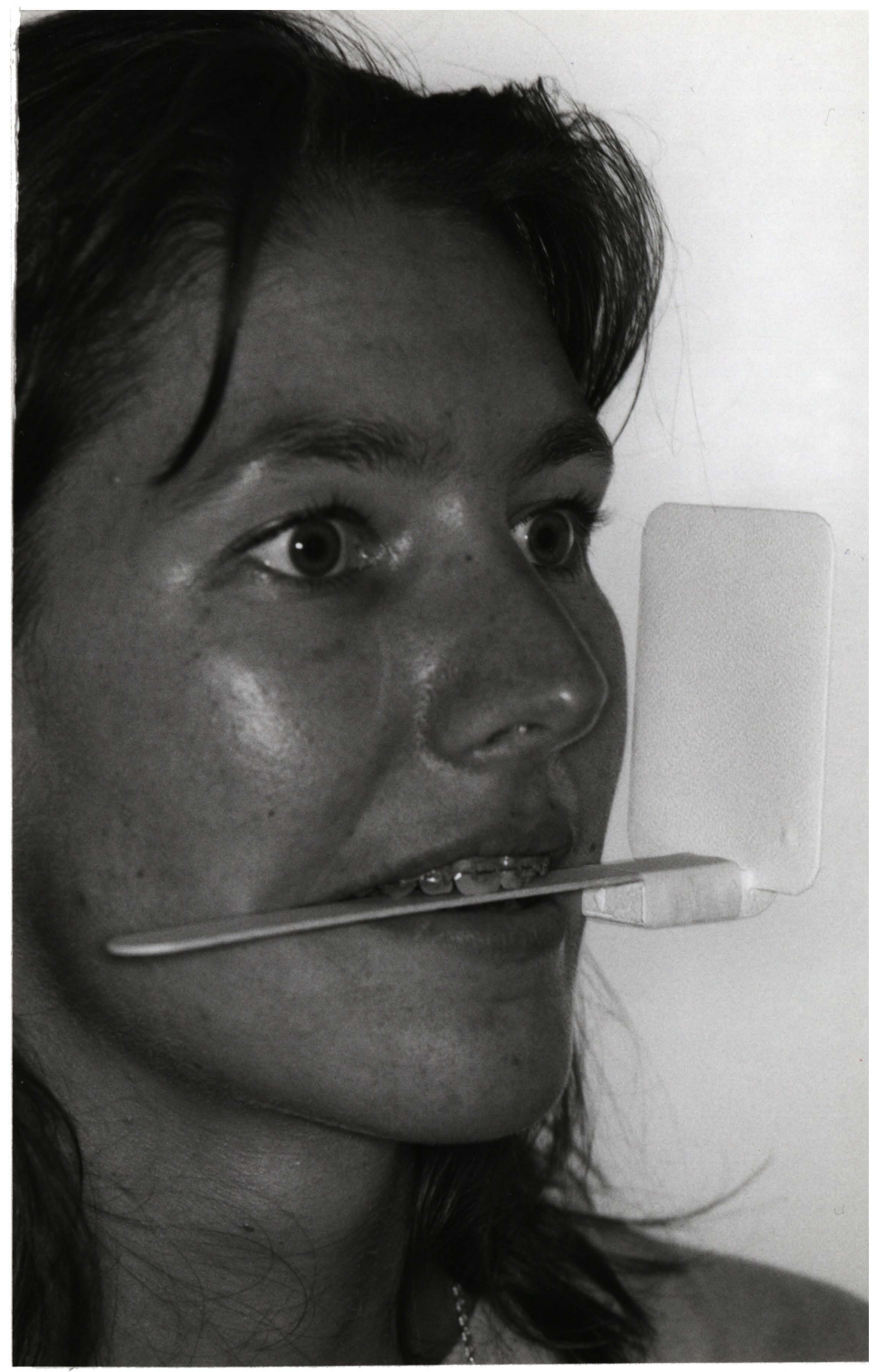




\section{Figure 6.}

The radiograph obtained shows the orientation of the vertical part of the wire to the long axis of the tooth. If the wire is not parallel it can be adjusted by removing it from the acrylic block and changing the bend. The horizontal part of the wire can be superimposed over the radiograph to check for parallelism between the vertical wire and the pulp canal.

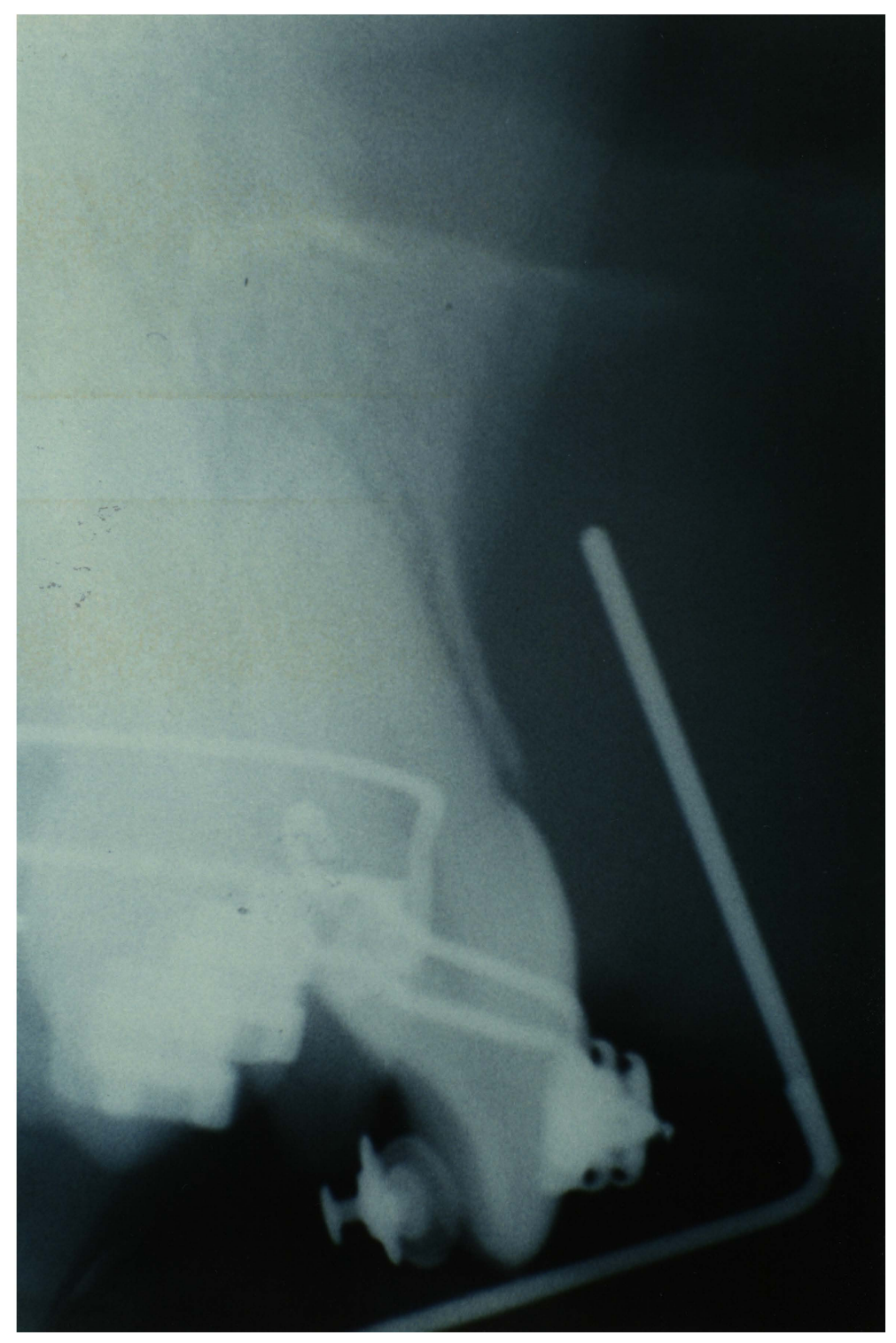




\section{Figure 7.}

If the wire element was removed it is reinserted in the acrylic. At this point a standard periapical film is taken.

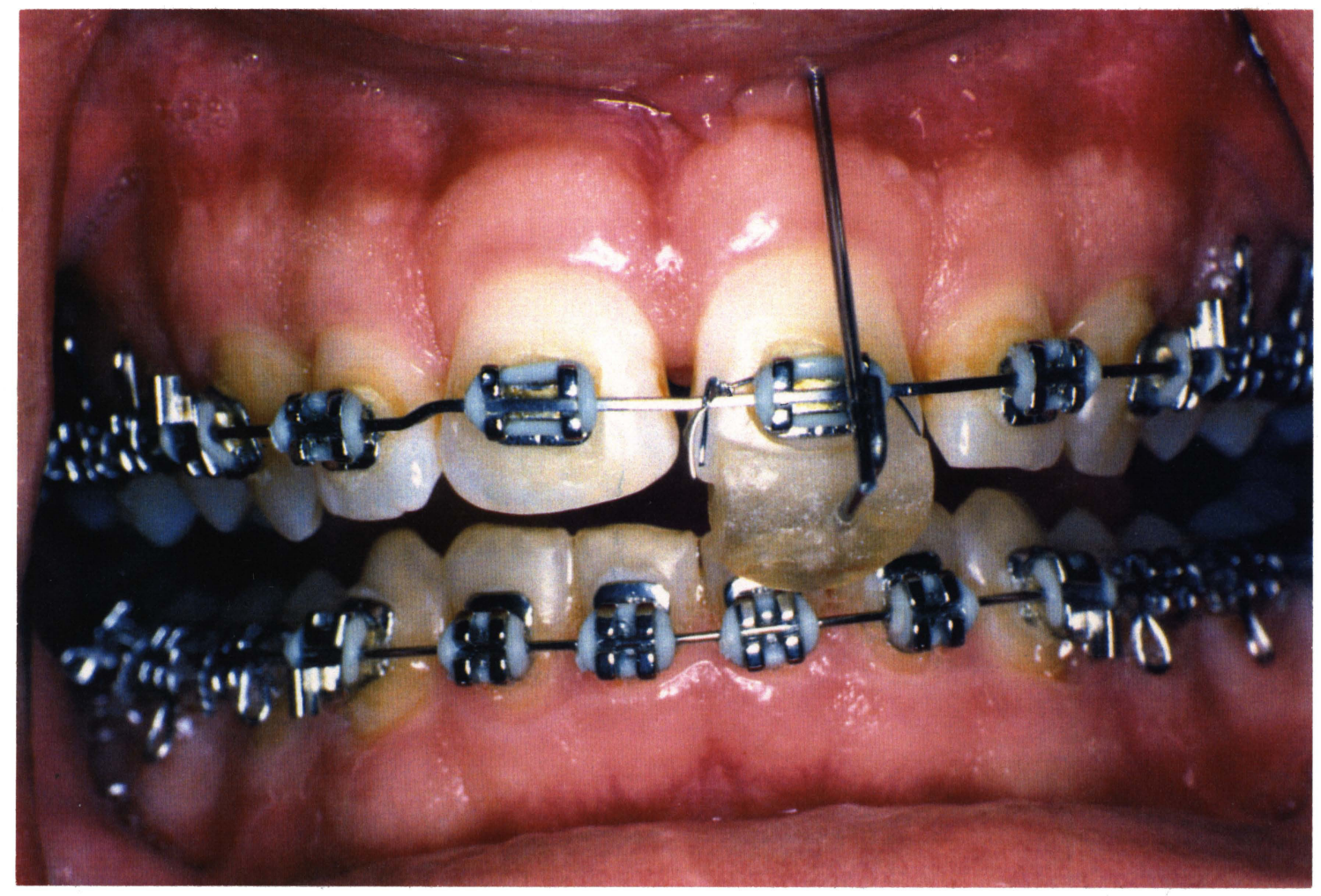




\section{Figure 8.}

Resulting radiograph with superimposed shadow of the wire. A precise magnification factor can now be calculated and used to find the actual length of the tooth.

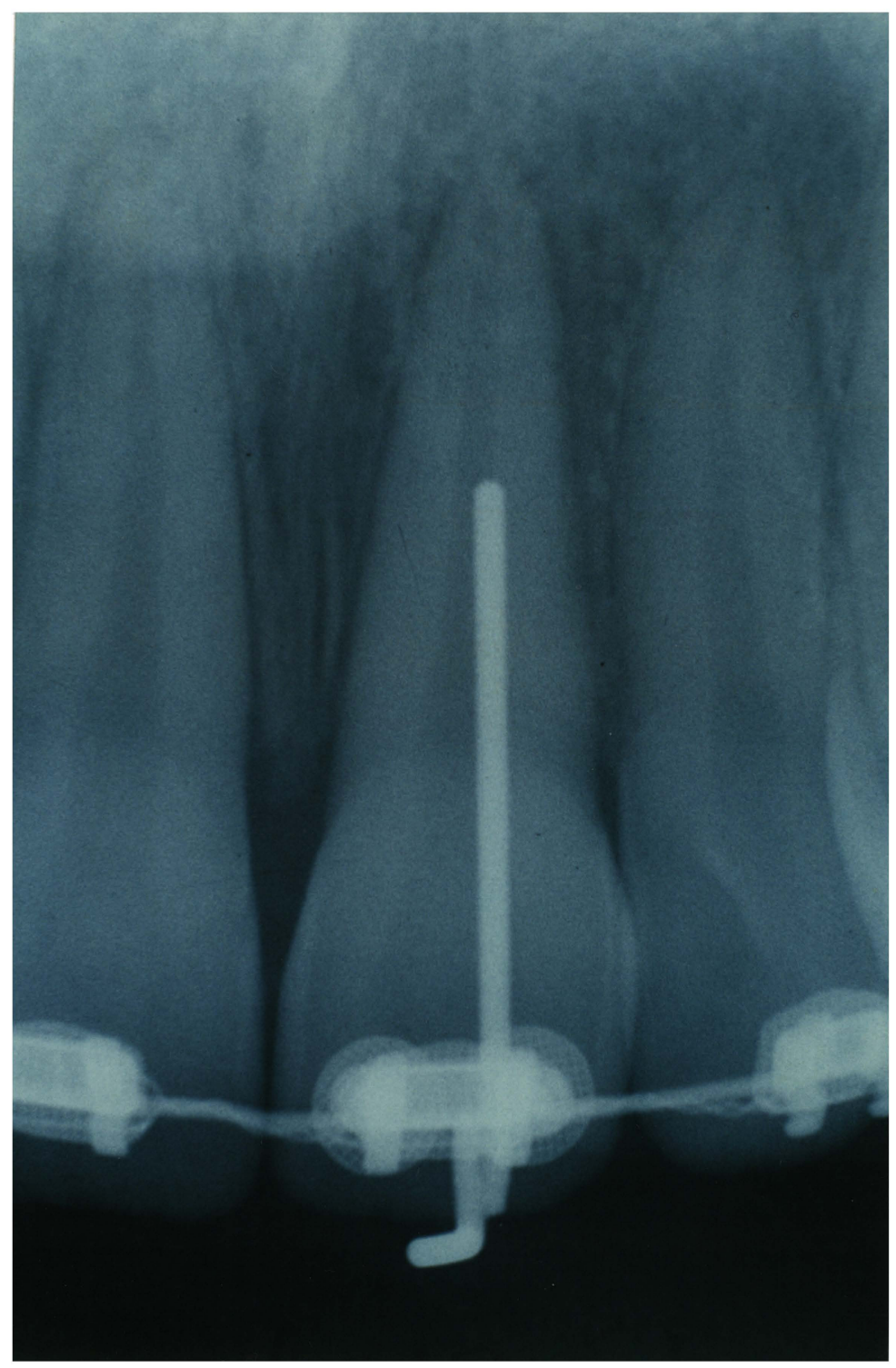


By comparing the true length of the wire to its dimension on the film, an exact magnification factor can be calculated for that exposure. Since the wire is registered to the crown via the acrylic block in a parallel orientation to the root, the magnification factor is valid for the tooth as well as the wire. This is due to the fact that parallel structures are foreshortened or elongated in the same proportion.

The overall length of the tooth on the film was measured along its long axis from apex to midpoint of the incisal edge. This radiographic measurement was then converted to actual length using the magnification factor found as described above. After determining actual tooth length at the time of the second radiograph using the same procedure, the change in length was calculated. All measurements were made using electronic calipers (accurate to $0.01 \mathrm{~mm}$ ).

To check reliability of the method, a test was conducted using 8 extracted maxillary incisors. The overall length of each tooth was measured with calipers and recorded. The teeth were then radiographed with a jig in place simulating the technique described above. Next, approximately $1.0 \mathrm{~mm}$ was ground off the apex of each tooth, the lengths were re-measured and recorded, and a second radiograph was taken. Using the radiographs taken before and after grinding, the change in length for each tooth was calculated per the method described above. The calculated values were compared to the actual amount removed by grinding.

Using the same before and after films of the eight teeth, the procedure for measuring resorption using crown/root ratios as described by Linge and Linge(9) and revised by Dermaut and De Munck(10) was also done. By this method the percent change in root lengths were found using the formula:

100 - root after $\times$ crown before $\times 100$ root before $\mathrm{x}$ crown after 
The percentages found were then multiplied by $16 \mathrm{~mm}$, the average root length for the 8 extracted teeth (measured on the interproximal surfaces).

When compared to the actual amounts that were ground off each tooth, the method of measurement using the wire jig had a mean error of $0.1 \mathrm{~mm}$ per tooth $(\mathrm{SD}=$ $0.1 \mathrm{~mm}$ ) and a coefficient of correlation $(r)=0.9$. The crown $/$ root ratio method had a mean error of $0.6 \mathrm{~mm}(\mathrm{SD}=0.4 \mathrm{~mm})$ and $r=0.4$. Based on these findings the newer method of using the jig to measure root shortening was employed for this clinical study.

Root resorption in a single central incisor for each patient was examined. $\mathrm{A} \mathrm{t}$ - test was done to compare the mean amount of resorption in the experimental and control groups. For the experimental group, coefficients of correlation were calculated for the relationship between resorption and each of the measured changes in tooth position. 


\section{Results}

For the experimental subjects, intrusion was carried out for a mean duration of 4.6 months (range 2.7 to 7.0 months). The average amount of intrusion was $1.9 \mathrm{~mm}$, measured at the center of resistance, and the mean rate of intrusion was $0.41 \mathrm{~mm}$ per month. Root resorption of the central incisors in the experimental group averaged $0.6 \mathrm{~mm}(\mathrm{SD}=0.6 \mathrm{~mm})$. The control group had a mean resorption of $0.2 \mathrm{~mm}$ $(\mathrm{SD}=0.6 \mathrm{~mm})$ after an interval of 4.3 months. A two-tailed $\mathrm{t}$ - test indicated a statistically significant difference between the resorption means $(\mathrm{p}<0.05)$. A histogram showing the distribution of patients in each group based on amount of root resorption is illustrated in Figure 6. Descriptive statistics for the two groups are given in Table I. and full data on each subject is listed in Appendix I.

The coefficient of correlation $(r)$ between the amount of resorption and the duration of treatment was $0.3(\mathrm{p}<0.05)$ for the intrusion group and 0.04 (not significant) for the control patients. Additionally, for the intrusion group a significant correlation was found between resorption and two of the measures of tooth movement: movement of the apex- $r=0.45(p<0.01)$, and vertical change of the incisal edge- $r=0.34(p<0.01)$. The correlations between resorption and intrusion, proclination, or AP change of the incisal edge were not significant. 

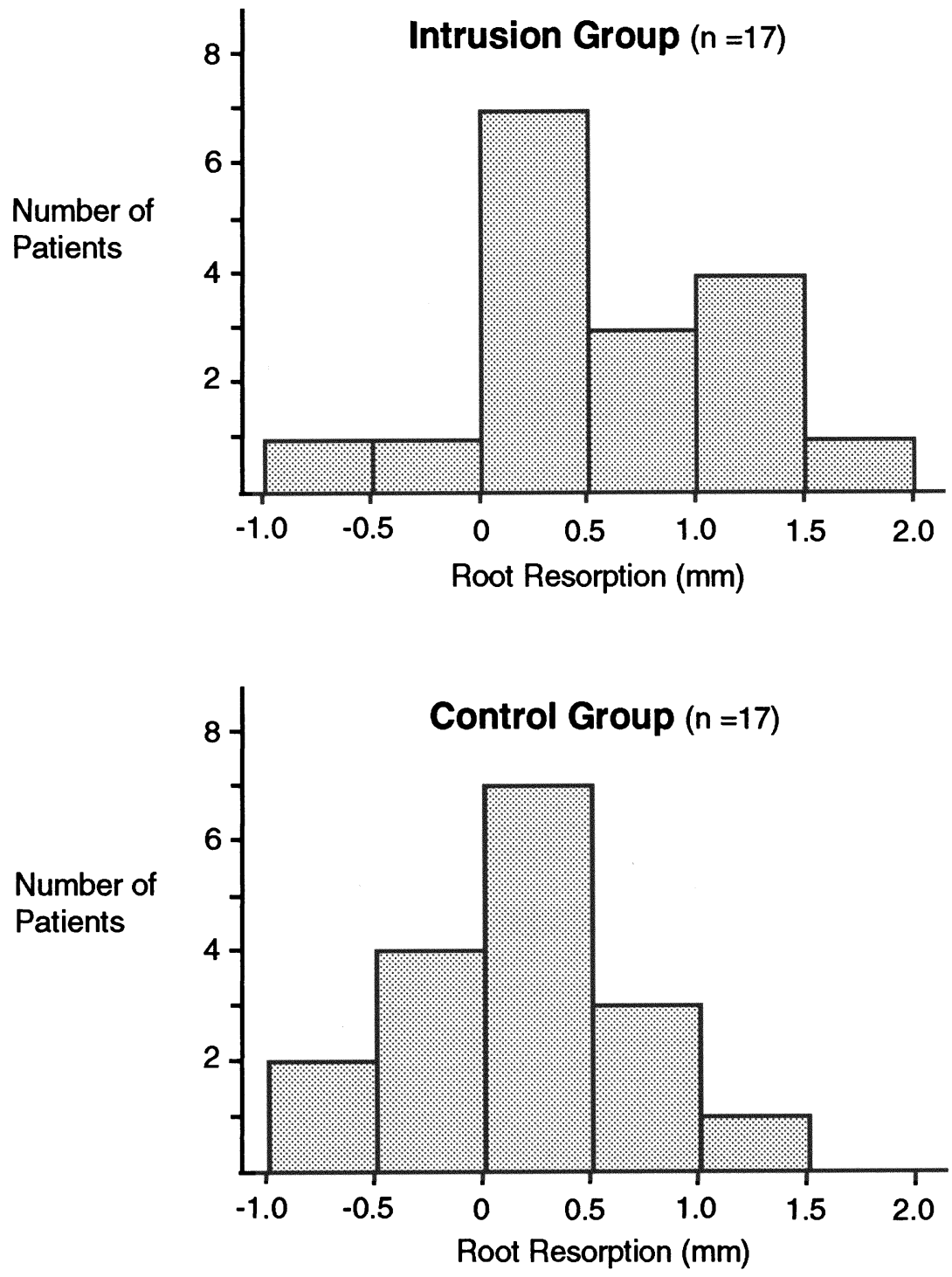

Figure 6. Distribution of patients based on amount of root resorption of the maxillary central incisor. (Negative values indicate the tooth measured longer after the period of treatment observed.) 


\begin{tabular}{|c|c|c|}
\hline Variable & Mean & SD \\
\hline \multicolumn{3}{|l|}{ Control Group $(n=17)$} \\
\hline Root Resorption (mm) & $0.2^{*}$ & 0.6 \\
\hline Time Interval (months) & 4.3 & 0.7 \\
\hline \multicolumn{3}{|l|}{ Experimental Group $(n=17)$} \\
\hline Root resorption (mm) & $0.6^{*}$ & 0.6 \\
\hline Time interval (months) & 4.6 & 1.3 \\
\hline Intrusion of CR (mm) & 1.9 & 0.8 \\
\hline Vertical change of incisal edge $(\mathrm{mm})$ & 2.3 & 0.8 \\
\hline AP change of incisal edge (mm) & 0.8 & 2.4 \\
\hline Proclination (degrees) & 7 & 7 \\
\hline Movement of apex (mm) & 3.0 & 1.0 \\
\hline
\end{tabular}

Table I. Changes in root length and tooth position of the maxillary central incisor.

Control subjects were random selection of patients in full arch appliances. Experimental subjects were treated with a maxillary intrusion arch. 


\section{Discussion}

A considerable amount of literature has been published on the relationship of orthodontics to root resorption. Investigators have examined factors relating to the patient, such as age, sex, root form, systemic conditions, type of malocclusion, etc.,

as well as factors in the orthodontic treatment, such as duration of treatment, characteristics of the tooth movement, type of appliance, and force levels. A recent extensive review by Brezniak and Wasserstein(12) highlights the fact that the methodology, sample selection, and results of these studies show considerable variation and do not allow many definite conclusions.

As a starting point, the present study sought to improve the means of quantifying apical root resorption. The method developed was tested using extracted teeth and changes in root length were measured with an average error of only 0.1 $\mathrm{mm}$ per tooth. Clinical application of this technique proved to be accurate and reasonably simple to use. The average amounts of root resorption for the experimental and control groups were found to differ by $0.4 \mathrm{~mm}$, which was statistically significant. The rate of resorption observed in the control group was an additional indication of the reliability of the methods employed. On average, these patients showed $0.2 \mathrm{~mm}$ of resorption after 4.3 months. Projected over a period of 24 months (typical length of treatment), this rate of apical resorption would lead to a loss of $1.1 \mathrm{~mm}$, which is consistent with published data.

The results of this study can be appropriately compared with those of Goerigk et al.(15) and Dermaut and De Munck(10). The mechanics used were very similar in all three studies. In terms of mean values, Goerigk et al.(15) found $0.9 \mathrm{~mm}$ of resorption of the central incisors after 4.3 months and a $2.3 \mathrm{~mm}$ vertical movement of the 
incisal edge, while patients in the present study had $0.6 \mathrm{~mm}$ of resorption after 4.6 months and $2.3 \mathrm{~mm}$ of movement (1.9 $\mathrm{mm}$ of intrusion measured at $\mathrm{CR}$ ).

Dermaut and De Munck(10), however, found $2.9 \mathrm{~mm}$ of resorption after 6.7 months and $3.6 \mathrm{~mm}$ of intrusion (of CR). The significantly greater amount of resorption reported by these authors is partly explainable by the duration of time that intrusion was performed (almost 50\% longer than the present study). But, given the low correlations found between root resorption and duration or extent of intrusion (by Dermaut and De Munck as well as in the present study), other factors may be responsible for the larger extent of resorption. One such factor could be the intrusive force levels used by Dermaut and De Munck were somewhat higher- 25 gm per tooth versus $15 \mathrm{gm}$ per tooth in the present study and that of Goerigk et al.(15). It is daunting to consider that root resorptive processes might be sensitive to force differences on the order of $10 \mathrm{gm}$ per tooth. Perhaps a critical force threshold exists beyond which the normal protective role of the periodontal ligament at the apex breaks down.

An interesting finding of the present study was the moderate correlation found between root resorption and movement of the apex of the tooth $(r=0.45)$. It would appear logical that the greater the distance the apex has to travel through bone, the longer the time it is in close proximity to inflammatory processes including osteoclastic activity. This conclusion is also supported by studies which have found a correlation between resorption and large buccal/lingual root movement(6) or the use of rectangular wires which produce torque(9). A correlation between root resorption and movement of the apex tends to contraindicate the practice of "round tripping", such as flaring incisors for the sake of alignment then following with space closure which tends to tip the teeth back again. 
The nature of the control group was a unique aspect of this study. By comparing root resorption in a random sample orthodontic patients to patients who received intrusion, an estimate of the additional resorption due to intrusion was possible. The patients who received intrusion had on average $0.4 \mathrm{~mm}$ more resorption than the "average" patient. It is unlikely that the small amount of additional resorption presumably caused by intrusion has any clinical impact.

Intrusion of incisors is an important treatment modality, being relatively stable and frequently the treatment of choice for the correction of excessive overbite(20). Opening the bite by extrusion of posterior teeth with bite plates, reverse curve wires, or cervical headgear is often contraindicated. Cases with low mandibular plane angles and strong musculature tend to relapse after extrusion, while high angle Class II cases can not afford to be hinged open. Additionally, excessive lip to tooth dimension can not be improved with posterior extrusion(16).

Perhaps the complex field of osteoclast biology will someday lead to pharmacologic regimens for the prevention of root resorption(21). More immediate promise seems to lie in studies that examine orthodontic methodology in an attempt to shed light on which forms of mechanotherapy lead to increased risk of resorption. In this study, the method used for intrusion was found to be effective in reducing overbite while causing only a small amount of root resorption, and rather less than some previous studies. 


\section{References}

1. Ketcham AH. A progress report of an investigation of apical root resorption of vital permanent teeth. Int J Orthod, Oral Surg, and Radiol. 1929;15:310.

2. Phillips JR. Apical root resorption under orthodontic therapy. Angle Orthod $1955 ; 25: 1-22$.

3. DeShields RW. A study of root resorption in treated Class II, Div I malocclusions. Angle Orthod 1969;39:231-245.

4. Goldson L, Henrikson CO. Root resorption during Begg treatment: a longitudinal roentgenographic study. Am J Orthod 1975;68:55-66.

5. Harris EF, Baker WC. Loss of root length and crestal bone height before and during treatment in adolescent and adult orthodontic patients. Am J Orthod Dentofac Orthop 1990;98:463-69.

6. Kaley J, Phillips C. Factors related to root resorption in edgewise practice.Angle Orthod 1991;61:125-132.

7. Levander E, Malmgren O. Evaluation of the risk of root resorption during orthodontic treatment: a study of upper incisors. Euro J Orthod 1988;10:30-38.

8. Linge BO, Linge L. Apical root resorption in upper anterior teeth. Euro J Orthod 1983;5:173-183.

9. Linge L, Linge BO. Patient characteristics and treatment variables associated with apical root resorption during orthodontic treatment. Am J Orthod Dentofac Orthop 1991;99:35-43.

10. Dermaut LR, DeMunck A. Apical root resorption of upper incisors caused by intrusive tooth movement: a radiographic study. Am J Orthod Dentofac Orthop 1986;90:321-326.

11. Harry MR, Sims MR. Root resorption in bicuspid intrusion- a scanning electron microscope study. Angle Orthod 1982;52:235-258. 
12. Bezniak N, Wasserstein A. Root resorption after orthodontic treatment. Am J Orthod Dentofac Orthop 1993;103:62-66, 138-146.

13. Caputo AA, Standlee JP. Biomechanics In Dentistry. Chicago: Quintessence Pub Co, 1987:86-87.

14. McFadden WM, Engstrom C, Engstrom H, Anholm JM. A study of the relationship between incisor intrusion and root shortening. Am J Orthod Dentofac Orthop 1989;96:390-396.

15. Goerigk B, Diedrich $\mathrm{P}$, Wehrbein H. Intrusion of the anterior teeth with the segmented arch technique of Burstone - a clinical study. [German] Fortschritte der Kieferorthopadie. 1992;53:16-25.

16. Burstone CR. Deep bite correction by intrusion. Am J Orthod 1977;72:1-22.

17. Harris EF, Butler ML. Patterns of incisor root resorption before and after orthodontic correction in cases with anterior open bites. Am J Orthod Dentofac Orthop 1992;101:112-19.

18. Newman WG. Possible etiologic factors in external root resorption. Am J Orthod $1955 ; 67: 522-39$

19. Remington DN, Joondeph DR, Artun J, Riedel RA, Chapko MK. Long-term evaluation of root resorption occurring during orthodontic treatment. Am J -Orthod Dentofac Orthop 1989;96:43-6.

20. Burzin J, Nanda R. The stability of deep overbite correction. In: Nanda R, Burstone C, ed. Retention and stability in orthodontics. Philadelphia: WB Saunders, 1993:61-79.

21. Andreasen JO. Summary of root resorption. In: Davidovitch Z, ed. The biological mechanisms of tooth eruption and root resorption. Birmingham: EBSCO Media. 1988:399-401. 
Appendix 1. Data for all subjects. Negative values for AP change and proclination indicate movement in the lingual direction. Negative values for root resorption indicate the tooth measured longer after the time interval. Movement of the apex was measured without respect to direction.

$\begin{array}{cccccccc}\text { Subject } & \begin{array}{c}\text { Time Interval } \\ \text { (months) }\end{array} & \begin{array}{c}\text { Root } \\ \text { Resorption } \\ \text { (millimeters) }\end{array} & \begin{array}{c}\text { Intrusion of CR } \\ \text { (millimeters) }\end{array} & \begin{array}{c}\text { Vertical Change } \\ \text { of Incisal Edge } \\ \text { (millimeters) }\end{array} & \begin{array}{c}\text { AP Change } \\ \text { of Incisal Edge } \\ \text { (millimeters) }\end{array} & \begin{array}{c}\text { Proclination } \\ \text { (degrees) }\end{array} & \begin{array}{c}\text { Movement } \\ \text { of Apex } \\ \text { (millimeters) }\end{array}\end{array}$

\section{Experimental}

$\begin{array}{rrrrrrrr}1 & 3.9 & 1.59 & 3.15 & 4.13 & 0.25 & 10 & 4.80 \\ 2 & 6.5 & 1.27 & 2.66 & 2.66 & 0.20 & 0 & 2.38 \\ 3 & 4.0 & 1.23 & 1.31 & 2.30 & 3.00 & 13 & 2.20 \\ 4 & 6.2 & 1.23 & 1.35 & 2.50 & -1.60 & 10 & 4.55 \\ 5 & 7.0 & 1.13 & 1.31 & 2.01 & -0.80 & 3 & 2.72 \\ 6 & 3.8 & 0.98 & 0.48 & 1.36 & 2.40 & 14 & 3.65 \\ 7 & 4.0 & 0.54 & * & * & * & * & * \\ 8 & 3.5 & 0.52 & 0.87 & 2.17 & 5.39 & 19 & 3.96 \\ 9 & 4.0 & 0.48 & 3.15 & 1.70 & -2.33 & -4 & 3.49 \\ 10 & 4.0 & 0.43 & 2.25 & 2.87 & -0.10 & 6 & 3.35 \\ 11 & 5.0 & 0.38 & 1.48 & 1.10 & -0.40 & -2 & 1.69 \\ 12 & 6.4 & 0.33 & 1.62 & 2.33 & 3.50 & 9 & 1.50 \\ 13 & 6.5 & 0.29 & * & * & * & * & * \\ 14 & 2.7 & 0.15 & 2.93 & 3.02 & -2.41 & 0 & 3.40 \\ 15 & 3.3 & 0.05 & 1.86 & 2.42 & 3.69 & 10 & 2.15 \\ 16 & 4.0 & -0.14 & * & * & * & * & * \\ 17 & 4.0 & -0.57 & 1.96 & 1.87 & -0.20 & 3 & 2.20 \\ & & & & & & & \end{array}$

\section{Control}

\begin{tabular}{|c|c|c|}
\hline 1 & 5.0 & 1.50 \\
\hline 2 & 3.6 & 0.88 \\
\hline 3 & 4.0 & 0.78 \\
\hline 4 & 4.2 & 0.60 \\
\hline 5 & 3.6 & 0.48 \\
\hline 6 & 4.7 & 0.32 \\
\hline 7 & 3.6 & 0.20 \\
\hline 8 & 4.9 & 0.15 \\
\hline 9 & 4.3 & 0.15 \\
\hline 10 & 4.7 & 0.08 \\
\hline 11 & 3.7 & 0.06 \\
\hline 12 & 5.9 & 0.00 \\
\hline 13 & 3.7 & -0.06 \\
\hline 14 & 3.7 & -0.16 \\
\hline 15 & 4.4 & -0.49 \\
\hline 16 & 3.6 & -0.69 \\
\hline 17 & 4.7 & -0.85 \\
\hline mean & 4.3 & 0.2 \\
\hline$S D$ & 0.7 & 0.6 \\
\hline
\end{tabular}

